\title{
Multiple Cutaneous and Uterine Leiomyomatosis
}

\author{
Teruhiko Makino
}

Additional information is available at the end of the chapter

http://dx.doi.org/10.5772/53601

\section{Introduction}

Multiple cutaneous and uterine leiomyomatosis (MCUL: OMIM 150800), which is also known as Reed syndrome, is an autosomal dominant disorder in which benign skin tumors arising from the arrector pili muscle and uterine fibroids typically develop in the third and fourth decades [1, 2]. Reed et al first reported on two families in which members of successive generations demonstrated cutaneous leiomyomas, uterine leiomyomas, and/or leiomyosarcomas in 1973 [3]. A small population of families with MCUL has also been reported to demonstrate clusters of renal cancer, either manifesting as type 2 papillary renal cell carcinoma or renal collecting duct cancer. This latter disease variant is referred to as hereditary leiomyomatosis and renal cell cancer (HLRCC: OMIM 605839) [4, 5]. Heterozygous germline mutations in the fumarate hydratase (FH, fumarase) gene (MIM 136850) mapped on chromosome 1q42.3-q43 are detected in both MCUL and HLRCC and many different mutations have been reported in the $F H$ gene $[6,7]$. The $F H$ gene encodes the fumarate hydratase $(\mathrm{FH})$ enzyme, that catalyzes the conversion of fumarate to malate as part of the TCA cycle in the mitochondrial matrix. This chapter will initially explain the clinical manifestations and etiology of MCUL/HLRCC based on the data from previous reports. The structure and fundamental function of the $\mathrm{FH}$ protein, $\mathrm{FH}$ gene mutation and the relation between alteration of FH protein and tumorigenesis in MCUL/HLRCC will be addressed. Finally, the diagnosis and treatments of MCUL/HLRCC is also explained.

\section{Clinical manifestations}

\subsection{Cutaneous leiomyomas}

The most prominent feature of MCUL/HLRCC is the occurrence of solitary or multiple cutaneous leiomyomas, which appear as firm skin-colored or pink-brown papules or nodules up to $2 \mathrm{~cm}$ in diameter and are often associated with pain (Figure 1) [1]. The distribution of skin lesions shows approximately equal numbers of patients with clustered leiomyomas only, 
scattered leiomyomas only, and a combination of clustered and scattered lesions. Clustered lesions are most common on the trunk, followed by the lower limb(s), upper limb(s), and head and neck. Scattered lesions are most often found on the the upper limb(s), followed by the trunk, lower limb(s), and head and neck. A small proportion of patients have symmetrically distributed or unilaterally distributed lesions. In addition, band-like or type 2 segmental manifestations have also been reported [8]. Skin leiomyomas are reported to develop at a mean age of 24.1 years (median, 25 years; range, 9-45 years), although the mean ages of symptom onset and diagnosis are 31.4 and 36.6 years, respectively. These tumors seem to remain benign. Only two cases of skin leiomyosarcoma in association with an $F H$ germline mutation have been reported $[9,10]$. A histological examination shows that all cutaneous leiomyomas are pilar lesions occurring superficially in the dermis (Figure 2). They were thought to originate from the pili arrector muscles of the hair follicle. Smooth muscle fiber bundles composed of eosinophilic cytoplasm with elongated blunt-ended nuclei with little or no waviness are interspersed with collagen within the dermis [11]. An immunohistochemical study revealed the presence of markers of smooth muscle differentiation, such as desmin and actin (Figure 3). Estrogen and progesterone receptors are negative in cutaneous leiomyomas, although these are positive in uterine leiomyomas [12].

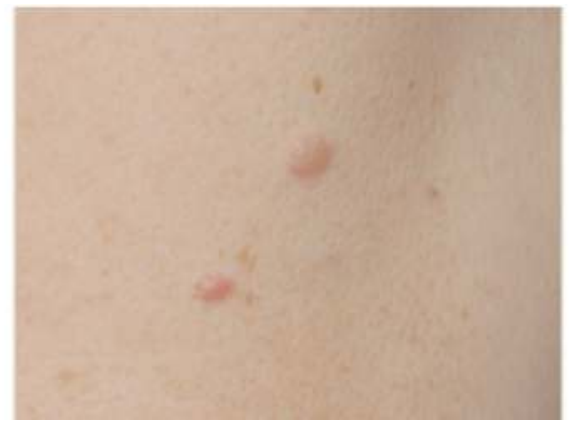

Figure 1. Clinical presentation of cutaneous leiomyoma. Redish nodules up to $2 \mathrm{~cm}$ in diametar.
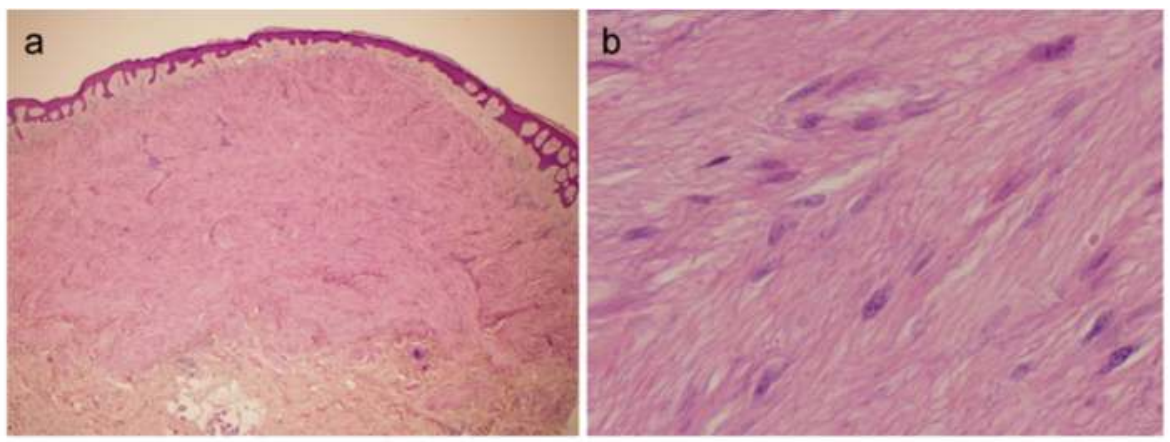

Figure 2. a. Histological examination of cutaneous leiomyoma shows interlacing fasicles of the smooth muscle cells within the dermis (hamtoxylin and eosin staining, original modification $x 40$ ). b. Tumor cells are composed of eosinophilic cytoplasm with wlogated blunt-ended nuclei. There were no atypia or mitosis present (hematoxylin and eosin staining, original modification $\times 400$ ) 


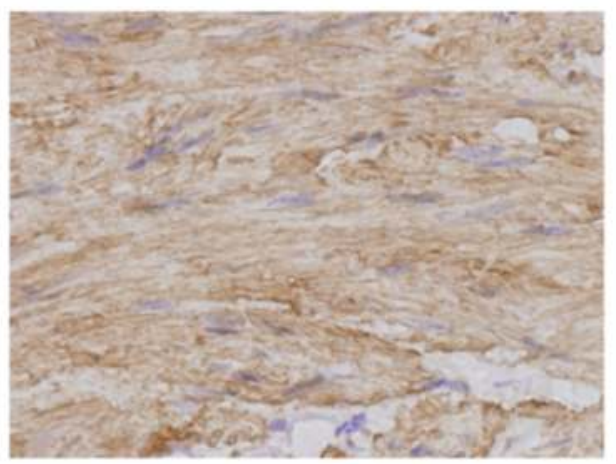

Figure 3. Immunohistological findings of cutaneous leiomyioma. Tumor cells were positive for smooth muscle actin immunostaining (original magnification $\times 400$ )

\subsection{Uterine fibroids}

Uterine fibroids (leiomyomas) are benign tumors that develop from the smooth muscle cells of the uterus. The common symptoms including irregular menses, menorrhagia, pain and defects in the reproductive functions, show no difference between uterine fibroids in MCUL/HLRCC and those of sporadic cases; however, the clinical features in MCUL/HLRCC are different from those in sporadic cases. Many uterine fibroids are observed in MCUL/HLRCC and the size of tumors in MCUL/HLRCC is larger than that of sporadic cases $[9,10]$. The mean age at the time of diagnosis of uterine fibroids with MCUL/HLRCC is around 30 years (range 18-53) and it is approximately 10 years before the diagnosis in sporadic cases. Most female patients (79-100\%) with an FH gene mutation are affected with uterine fibroids $[10,13]$. The association of the generally rare uterine leiomyosarcoma with the syndrome has also been suggested; however, the biological behavior of the uterine tumors in HLRCC has remained unclear [14].

\subsection{Renal cell carcinoma}

Renal cell carcinoma (RCC) is a tumor arising from the epithelium of the renal tubules. RCC can be classified into morphological subtypes including clear cell, papillary, chromophobe and collecting duct carcinoma $[15,16]$. The most frequent type of RCC in HLRCC is a type 2 papillary RCC [17]. The tumor histologically shows a papillary growth pattern. The tumor cells show a large nucleus with a prominent eosinophilic nucleus surrounded by a clear halo. Cystic components also seem to be typical findings [18, 19]. These features are suggested to be characteristic of a RCC in HLRCC. In addition, collecting duct tumors, oncocytic tumors and clear cell tumors have also been reported [9, 10, 18, 20]. An immunohistochemical study of RCC in HLRCC showed the absence of the cytokeratin (CK) 7 and the expression of UEA-a protein. In addition, the absence of mucin, CK20 and CD10 is considered to be typical of the tumors $[19,21]$. RCC in HLRCC is commonly solitary and unilateral. RCC is found in about $20-25 \%$ of the $F H$ gene mutation positive families [22, 23]. 
Up to 32 and $50 \%$ of the North America and Finnish families, respectively, show the RCC phenotype $[9,10,18]$ The mean age at the time of RCC diagnosis is 42 and 44 years in Finnish and North American HLRCC families, respectively. Approximately half cases of the RCC in HLRCC are detected in individuals younger than 40 years, many even less than 30 years [9, 20, 24]. The youngest of the RCC patients was 11 years old when diagnosed [24]. Importantly, HLRCC-associated renal cancers are very aggressive and can metastasize even though the primary tumor is small. Most reported patients die within 5 years after diagnosis creating a challenge for surveillance and treatment practices $[9,19,25]$. Therefore, annual pelvic/abdominal MRI starting from the age of 18 is considered to be effective practice, especially for individuals with a familial history of RCC. In addition, benign kidney cysts frequently develop in the carriers of $F H$ gene mutation in comparison to individuals less than 40 years of age in the general population. Such cyst formation is suggested to result from an increased cell proliferation due to the activation of the hypoxia pathway and it has also been postulated to represent premalignant lesions [26, 27].

\subsection{Fumarate hydratase}

FH gene is located in the chromosomal region 1q42.1. It contains 10 exons and generates a transcript of $1.5 \mathrm{~kb}[28,29]$. There is a mitochondria localization signal in the first exon. $\mathrm{FH}$ gene encodes two isoforms of the fumarate hydratase $(\mathrm{FH})$ enzyme. The mitochondrial isoform of $\mathrm{FH}$ is one of the enzymes of the tricarboxylic acid cycle (TCA cycle, Kreb's cycle), which is a part of cellular respiration, the aerobic step of energy production (Figure 4). The active form of FH protein is a homotetramer with two substrate-binding sites [13] and it catalyzes the conversion of fumarate to malate in the mitochondrial matrix. In contrast, the function of the cytosolic FH isoform is thought to be involved in the fumarate and amino acid metabolism [30]. Previous studies suggest that some of the FH protein is translocated back to the cytosol from the mitochondria by removal of the mitochondrial localization signal [31].

Heterozygous germline mutations in FH gene were linked with both MCUL and HLRCC [6, 32]. Biallelic inactivation of FH is observed in associated tumors; therefore, $\mathrm{FH}$ is considered to be a tumor suppressor based on the Knudsen's two-hit hypothesis.

\subsection{Mutations in $\mathrm{FH}$ gene}

Approximately 100 different mutations have been reported in the $F H$ gene according to the online FH variant database [22]. Missense, nonsense, frameshift, insertion, and splice-site mutations have been found in the $\mathrm{FH}$ gene. The majority ( $58 \%)$ of these germline mutations are missense with the remaining being nonsense $(\sim 11 \%)$, and frameshift $(\sim 11 \%)$ mutations, located along the entire length of the $F H$ gene coding region. Nonsense mutations result in the absence of $\mathrm{FH}$ or formation of a truncated $\mathrm{FH}$ protein product that is functionally inactive. Most of the heterozygous missense mutations are found in the looped regions of $\mathrm{FH}$ with important roles in forming the homotetramer according to the crystal structure of the E.coli fumarase C, which is useful in models for predicting the effect of missense 


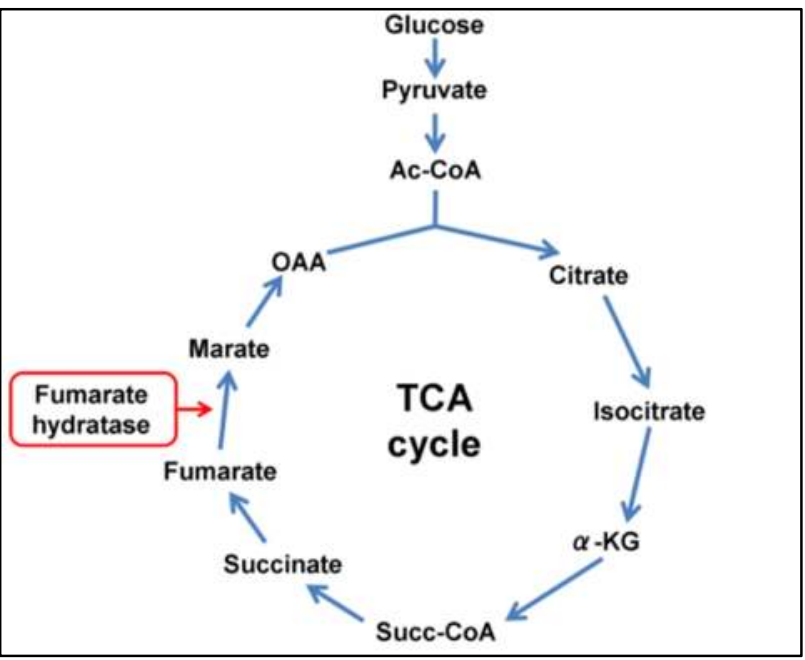

Figure 4. TCA cycle. Ac-CoA, acetyl coenzyme A; $\alpha$-KG, $\alpha$-ketoglurarase; Succ-CoA, succinyl coenzyme A; OOA, oxaloacetate.

mutations on human FH [33]. These missense mutations paradoxically cause marked reduction in the $\mathrm{FH}$ enzyme activity in comparison to truncated mutations [8, 13]. In addition, a hypothesis of the dominant negative effect of missense mutations has also been reported [34]. There appears to be no specific genotype-phenotype correlation with regard to which combination of these tumors develops in MCUL/HLRCC [32]. However, cases with RCC in HLRCC are mainly found in Finnish and North American families. This suggests that either environmental or additional genetic factors might be related to the induction of the malignant phenotype $[2,4-6,10,18]$. Several other tumors have also been reported In the $\mathrm{FH}$ gene mutation carriers. However, biallelic inactivation of the $F H$ gene was detected in only three cases of breast cancer, one case of bladder cancer, two cases of adult Leydig cell tumors and one case of adrenocortical hyperplastic lesion in Cushing syndrome [18, 35, 36]. The significance of $F H$ gene mutation in the development of these tumors is still unclear although the $F H$ gene defect might be involved in the tumorigenesis. In addition, biallelic $\mathrm{FH}$ germline mutations cause a rare recessive syndrome named $\mathrm{FH}$ deficiency (FHD or fumaric aciduria, MIM 606813), characterized by severe neurological symptoms such as psychomotor retardation, muscular hypotonia and microcephaly [37, 38].Dramatic reduction of the $\mathrm{FH}$ enzyme activity in a patient's tissues results in a metabolic crisis causing death commonly as an infant.

\subsection{Molecular mechanism of MCUL/HLRCC tumorigenesis}

Individuals with MCUL/HLRCC inherit one loss-of function allele and somatically lose the other allele in the tumor. The inherited $F H$ gene mutations severely reduce enzyme activity, causing the tumors to accumulate high levels of fumarate [10]. Both loss of heterozygosity $(\mathrm{LOH})$ and point mutations as second hits have been observed as this mechanism $[5,39]$. 
Therefore, the $F H$ gene is thought to act as a tumor suppressor gene [4]. However, the $F H$ gene is not a typical tumor suppressor gene with a distinct anti-proliferative role, but rather its loss leads to more complex consequences. One of the broadly studied mechanisms is the so-called "pseudohypoxia" pathway referring to the induction of the hypoxia inducible factor 1 (HIF1) and its downstream targets under normoxic condition. HIF1 is a heterodimeric transcription factor formed by the HIF1 $\alpha$ and HIF1 $\beta$ subunit. The proteosomal degradation of the HIF1 $\alpha$ subunit is important for HIF1 regulation when molecular oxygen is available. HIF1 promotes adaptation of cells to non-physiological conditions when oxygen tension is low, by inducing anaerobic glycolysis as an alternative phosphorylation, and by inducing vascularization to facilitate the oxygen and nutrient supply into hypoxic tissues [40]. Fumarate, the substrate of $\mathrm{FH}$, is shown to accumulate into the cytoplasm of cells and cause stabilization of HIF1 by inhibiting $\alpha$-ketoglutarate $(\alpha-\mathrm{KG})$ dependent dioxygenase in MUCL/HLRCC tumors because of an FH defect [41-44]. The stabilized HIF1 plays a role as an activator in vascularization, glycolysis and glucose transport, which are significant pathways for promoting tumor growth [45]. Furthermore, FH deficiency precludes tumor cells from generating several of the TCA cycle intermediates, including malate, oxaloacetate and citrate, through conventional oxidative metabolism. [46, 47]. Human FH-deficient renal carcinoma cells redirect part of the TCA cycle, to compensate for this. This pathway appears to be a robust mechanism allowing cells to maintain growth during impaired oxidative metabolism, because it is also observed in human cancer cell lines with a mutation in the electron transport chain or in the Von Hippel-Lindau tumor suppressor, and in cells subjected to hypoxia, all of which negatively impact oxygendependent mitochondrial enzymes [48-51]. In addition, high levels of fumarate can induce aberrant patterns of gene expression. The electrophilic properties of fumarate allow it to modify cysteine residues on cellular proteins, producing an S-(2-succinyl)cysteine adduct in a Michael addition reaction termed succination [52]. Succination impairs protein function. Fumarate-mediated succination of Kelch-like ECH-associated protein 1 (Keap1) elicits an nuclear factor E2-related factor 2 (Nrf2) response In cells with FH deficiency, maintaining constitutively high expression of Nrf2 targets [53-55]. One of these targets is HMOX1, which encodes the enzyme required for heme degradation in FH-deficient cells, thus suggesting that fumarate-dependent suppression of Keap1 may promote cell survival, although the role the Keap1/Nrf2 system plays in tumorigenesis is unclear. Therefore, fumarate-mediated suppression of Keap1 may contribute to tumor development in the setting of FH deficiency.

\subsection{Diagnosis}

No diagnostic criteria for MUUL/HLRCC have been established; however, practical criteria for the clinical diagnosis of MUUL/HLRCC have been proposed [56]. Multiple cutaneous leiomyoma which is histologically confirmed is proposed as a major criterion. The minor criteria included uterine fibroids, papillary type 2 RCC or positive familial history. A molecular genetic analysis should be conducted to confirm the diagnosis when the clinical features are suggestive of MCUL/HLRCC. Direct sequencing of the $F H$ gene cording region 
is commonly performed as a genetic analysis. This analysis detects underlying genetic alterations in about $90 \%$ of the suggestive MCUL/HLRCC cases [9, 10, 20, 57]. The possibility of exon or whole gene deletion is suspected If no mutation is detected in the $F H$ gene, in spite of the fact that either patient's symptoms or familial history is strongly suggestive of UCML/HLRCC. The detection of possible copy-number changes in the FH gene might be useful in such case, by using additional methods such as multiplex ligationdependent probe amplification $[56,58]$.

\section{Treatment and management}

\subsection{Cutaneous leiomyomas and uterine fibroids}

Cutaneous leiomyomas are commonly benign, and thus, the treatment for these tumors may be only improvement of cosmetic and pain related complications. Surgical excision is usually performed for the solitary tumors. The multiple painful lesions are generally treated with medications, such as nitoglycerol, calcium channel blockers, alpha-adrenoreceptor blockers, which have been reported to be occasionally successful to relieve pain [8]. Surgical approaches including hysterectomy are typically needed for uterine fibroids, based on the number and size of the tumors and the severity of the symptoms caused by the tumors [13, 57]. Furthermore, myomectomy, uterine artery embolization or pharmaceutical treatment with gonadotropin-releasing hormone agonist is also performed as an optional treatment for uterine fibroids.

\subsection{Renal cell carcinoma}

RCCs commonly acquires metastatic potential after exceeding the size of 3-7 cm [59]. Renal lesions can be observed until they reach the size of $3 \mathrm{~cm}$, at which point they should be removed, and nephron sparing surgery usually appropriate [60]. However, RCC in HLRCC is thought to differ from sporadic RCCs because they are often metastatic at presentation even if the size of tumor is less than $1 \mathrm{~cm}$. Therefore, tumors in HLRCC are recommended to be excised with radical surgery immediately $[15,59,60]$. Sorafenib and sunitinib, which are inhibitors of receptor tyrosine kinases activated by HIF1 targets such as VEGF, PDGF and TNF- $\alpha$, have been used in the treatment of sporadic papillary RCC with varying success. These treatments are specific targeted pharmaceutical approaches. However, Information regarding the specific response of HLRCC associated tumors to these molecules is not available.

\section{Conclusion}

MCUL/HLRCC is a syndrome predisposing the $F H$ gene mutation carriers mainly to benign tumors including cutaneous leiomyomas and uterine fibroids. Furthermore, renal cell carcinomas are also found in a subset of the HLRCC families and these are very aggressive in nature as small lesions. Therefore, appropriate surveillance with diagnostic examination 
for uterine and diseases is warranted in rare cases of multiple, biopsy-proven cutaneous lesions. Genetic analysis of the FH gene should be performed in all cases of suspected or confirmed disease. Genetic counseling is also recommended for other family members of the patient's family. Identification of the syndrome and its tumorigenic mechanisms has provided new insight in MCUL/HLRCC.

\section{Author details}

Teruhiko Makino

Department of Dermatology, Graduate School of Medicine and Pharmaceutical Sciences, University of Toyama, Toyama, Japan

\section{References}

[1] Garcia Muret MP, Pujol RM, Alomer A et al. Familial leiomyomatosis cutis et uteri (Reed's syndrome). Arch Dermatol Res 1988; 280: S29-32.

[2] Alam NA, Bevan S, Churchman M et al. Localization of a gene (MCUL1) for multiple cutaneous leiomyomata and uterine fibroids to chromosome 1q42.3-q43. Am J Hum Genet 2001; 68:1264-1269

[3] Reed WB, Walker R, Horowitz R. Cutaneous leiomyomata with uterine leiomyomata. Acta Derm Venereol 1973; 53:409-416

[4] Kiuru M, Launonen V, Hietala M et al. Familial cutaneous leiomyomatosis is a two-hit condition associated with renal cell cancer of characteristic histopathology. Am J Pathol 2001; 159: 825-829

[5] Launonen V, Vierimaa O, Kiuru M et al (2001) Inherited susceptibility to uterine leiomyomas and renal cell cancer. Proc Natl Acad Sci USA 98:3387-3392

[6] Tomlinson IP, Alam NA, Rowan AJ et al. Germline mutations in FH predispose to dominantly inherited uterine fibroids, skin leiomyomata and papillary renal cell cancer. Nat Genet 2002; 30: 406-410

[7] Badeloe S, van Geest AJ, van Marion AM et al. Absence of fumarate hydratase mutation in a family with cutaneous leiomyosarcoma and renal cancer. Int J Dermatol 2008; 47(Suppl 1): 18-20

[8] Ritzmann S, Hanneken S, Neumann NJ et al. Type 2 segmental manifestation of cutaneous leiomyomatosis in four unrelated women with additional uterine leiomyomas (Reed's syndrome). Dermatology 2006; 212: 84-87

[9] Toro JR, Nickerson ML, Wei MH et al. Mutations in the fumarate hydratase gene cause hereditary leiomyomatosis and renal cell cancer in families in North America. Am J Hum Genet 2003; 73:95-106

[10] Wei MH, Toure O, Glenn GM et al. Novel mutations in FH and expansion of the spectrum of phenotypes expressed in families with hereditary leiomyomatosis and renal cell cancer. J Med Genet 2006; 43: 18-27 
[11] Kilpatrick SE, Mentzel T, Fletcher CD. Leiomyoma of deep soft tissue. Clinicopathologic analysis of a series. Am J Surg Pathol 1994; 18: 576-582.

[12] McGinley KM, Bryant S, Kattine AA, et al. Cutaneous leiomyomas lack estrogen and progesterone receptor immunoreactivity. J Cutan Pathol 1997; 24: 241-245.

[13] Alam NA, Barclay E, Rowan AJ et al. Clinical features of multiple cutaneous and uterine leiomyomatosis: an underdiagnosed tumor syndrome. Arch Dermatol 2005; 141: 199-206

[14] D’Angelo E, Prat J. Uterine sarcomas: a review. Gynecol Oncol 2010; 116: 131-139

[15] Cohen HT, McGovern FJ. Renal-cell carcinoma. N Engl J Med 2005; 353:2477-2490

[16] Eble JN, Sauter G, Epstein JI, Sesterhenn IA (ed.) World Health Organization classification of tumours: pathology \& genetics of tumours of the urinary system and male genital organs. Lyon: IARC Press; 2004

[17] Delahunt B, Eble JN. Papillary renal cell carcinoma: a clinicopathologic and immunohistochemical study of 105 tumors. Mod Pathol 1997; 10: 537-544

[18] Lehtonen HJ. Molecular and clinical characteristics of tricarboxylic acid cycle-associated tumors. Dissertation: University of Helsinki; 2008

[19] Merino MJ, Torres-Cabala C, Pinto P et al. The morphologic spectrum of kidney tumors in hereditary leiomyomatosis and renal cell carcinoma (HLRCC) syndrome. Am J Surg Pathol 2007; 31: 1578-1585

[20] Alam NA, Rowan AJ, Wortham NC et al. Genetic and functional analyses of FH mutations in multiple cutaneous and uterine leiomyomatosis, hereditary leiomyomatosis and renal cancer, and fumarate hydratase deficiency. Hum Mol Genet 2003; 12: 1241-1252

[21] Lehtonen HJ, Kiuru M, Ylisaukko-Oja SK et al. Increased risk of cancer in patients with fumarate hydratase germline mutation. J Med Genet 2006; 43: 523-526

[22] Bayley JP, Launonen V, Tomlinson IP. The FH mutation database: an online database of fumarate hydratase mutations involved in the MCUL (HLRCC) tumor syndrome and congenital fumarase deficiency. BMC Med Genet 2008; 9: 20

[23] Koski TA. Molecular genetic background of tumours in hereditary leiomyomatosis and renal cell cancer syndrome. Dissertation: University of Helsinki; 2010

[24] Alrashdi I, Levine S, Paterson J et al. Hereditary leiomyomatosis and renal cell carcinoma: very early diagnosis of renal cancer in a paediatric patient. Fam Cancer 2010; 9: $239-243$

[25] Grubb RL 3rd, Franks ME, Toro J et al. Hereditary leiomyomatosis and renal cell cancer: a syndrome associated with an aggressive form of inherited renal cancer. J Urol 2007; 177: 2074-2079

[26] Pollard PJ, Spencer-Dene B, Shukla D et al. Targeted inactivation of fh1 causes proliferative renal cyst development and activation of the hypoxia pathway. Cancer Cell 2007; 11:311-319 
[27] Mandriota SJ, Turner KJ, Davies DR et al. HIF activation identifies early lesions in VHL kidneys: evidence for site-specific tumor suppressor function in the nephron. Cancer Cell 2002; 1: 459-468

[28] Kinsella BT, Doonan S. Nucleotide sequence of a cDNA coding for mitochondrial fumarase from human liver. Biosci Rep 1986; 6: 921-929

[29] Tolley E, Craig I. Presence of two forms of fumarase (Fumarate Hydratase E.C. 4.2.1.2) in mammalian cells: immunological characterization and genetic analysis in somatic cell hybrids. Confirmation of the assignment of a gene necessary for the enzyme expression to human chromosome 1. Biochem Genet 1975; 13: 867-883

[30] Weaver TM, Levitt DG, Donnelly MI, et al. The multisubunit active site of fumarase C from Escherichia coli. Nat Struct Biol 1995; 2: 654-662.

[31] Sass E, Karniely S, Pines O. Folding of fumarase during mitochondrial import determines its dual targeting in yeast. J Biol Chem 2003; 278: 45109-45116

[32] Badeloe S, van Geel M, van Steensel MA et al. Diffuse and segmental variants of cutaneous leiomyomatosis: novel mutations in the fumarate hydratase gene and review of the literature. Exp Dermatol 2006; 15: 735-741

[33] Alam NA, Olpin S, Rowan A, et al: Missense mutation in Fumarate Hydratase in Multiple Cutaneous and Uterine Leiomyomatosis and Renal Cell Cancer. J Mol Diagn 2005; 7: 437-443.

[34] Lorenzato A, Olivero M, Perro M et al. A cancer-predisposing "hot spot" mutation of the fumarase gene creates a dominant negative protein. Int J Cancer 2008; 122: 947-951

[35] Matyakhina L, Freedman RJ, Bourdeau I et al. Hereditary leiomyomatosis associated with bilateral, massive, macronodular adrenocortical disease and atypical Cushing syndrome: a clinical and molecular genetic investigation. J Clin Endocrinol Metab 2005; 90: 3773-3779

[36] Carvajal-Carmona LG, Alam NA, Pollard PJ et al. Adult leydig cell tumors of the testis caused by germline fumarate hydratase mutations. J Clin Endocrinol Metab 2006; 91: 3071-3075

[37] Zinn AB, Kerr DS, Hoppel CL. Fumarase deficiency: a new cause of mitochondrial encephalomyopathy. N Engl J Med 1986; 315: 469-475

[38] Deschauer M, Gizatullina Z, Schulze A et al. Molecular and biochemical investigations in fumarase deficiency. Mol Genet Metab 2006; 88: 146-152

[39] Kiuru M, Lehtonen R, Arola J et al. Few FH mutations in sporadic counterparts of tumor types observed in hereditary leiomyomatosis and renal cell cancer families. Cancer Res 2002; 62: 4554-4557

[40] Semenza GL. Oxygen homeostasis. Wiley Interdiscip Rev Syst Biol Med 2010; 2: 336-361

[41] Pollard PJ, Briere JJ, Alam NA et al. Accumulation of Krebs cycle intermediates and over-expression of HIF1alpha in tumours which result from germline $\mathrm{FH}$ and SDH mutations. Hum Mol Genet 2005; 14: 2231-2239 
[42] Isaacs JS, Jung YJ, Mole DR et al. HIF overexpression correlates with biallelic loss of fumarate hydratase in renal cancer: novel role of fumarate in regulation of HIF stability. Cancer Cell 2005; 8: 143-153

[43] MacKenzie ED, Selak MA, Tennant DA et al. Cell-permeating alpha-ketoglutarate derivatives alleviate pseudohypoxia in succinate dehydrogenase-deficient cells. Mol Cell Biol 2007; 27: 3282-3289

[44] Pan Y, Mansfield KD, Bertozzi CC et al. Multiple factors affecting cellular redox status and energy metabolism modulate hypoxia-inducible factor prolyl hydroxylase activity in vivo and in vitro. Mol Cell Biol 2007; 27: 912-925

[45] Ashrafian H, O'Flaherty L, Adam J et al. Expression profiling in progressive stages of fumarate-hydratase deficiency: the contribution of metabolic changes to tumorigenesis. Cancer Res 2010; 70: 9153-9165

[46] Shanware NP, Mullen AR, DeBerardinis RJ et al. Glutamine: pleiotropic roles in tumor growth and stress resistance. J Mol Med (Berl) 2011; 89: 229-236

[47] DeBerardinis RJ, Cheng T. Q's next: the diverse functions of glutamine in metabolism, cell biology and cancer. Oncogene 2010; 29: 313-324

[48] Wise DR, Ward PS, Shay JE et al. Hypoxia promotes isocitrate dehydrogenasedependent carboxylation of alpha-ketoglutarate to citrate to support cell growth and viability. Proc. Natl. Acad. Sci. U.S.A. 2011; 108: 19611-19616

[49] Mullen AR, Wheaton WW, Jin ES et al. Reductive carboxylation supports growth in tumour cells with defective mitochondria. Nature 2012; 481: 385-388

[50] Metallo CM, Gameiro PA, Bell EL et al. Reductive glutamine metabolism by IDH1 mediates lipogenesis under hypoxia. Nature 2012; 481: 380-384

[51] Scott DA, Richardson AD, Filipp FV et al. Comparative metabolic flux profiling of melanoma cell lines: beyond the Warburg effect. J Biol Chem 2011; 286: 42626-42634

[52] Alderson NL, Wang Y, Blatnik M et al. S-(2-Succinyl) cysteine: a novel chemical modification of tissue proteins by a Krebs cycle intermediate. Arch Biochem Biophys 2006; 450: $1-8$

[53] Ooi A, Wong JC, Petillo D et al. An antioxidant response phenotype shared between hereditary and sporadic type 2 papillary renal cell carcinoma. Cancer Cell 2011; 20: 511523

[54] Adam J, Hatipoglu E, O'Flaherty L et al. Renal cyst formation in Fh1-deficient mice is independent of the Hif/Phd pathway: roles for fumarate in KEAP1 succination and Nrf2 signaling. Cancer Cell 2011; 20: 524-537

[55] Bardella C, El-Bahrawy M, Frizzell N et al. Aberrant succination of proteins in fumarate hydratase-deficient mice and HLRCC patients is a robust biomarker of mutation status. J Pathol 2011; 225: 4-11

[56] Smit DL, Mensenkamp AR, Badeloe S et al. Hereditary leiomyomatosis and renal cell cancer in families referred for fumarate hydratase germline mutation analysis. Clin Genet 2011; 79: 49-59 
[57] Stewart L, Glenn GM, Stratton P et al. Association of germline mutations in the fumarate hydratase gene and uterine fibroids in women with hereditary leiomyomatosis and renal cell cancer. Arch Dermatol 2008; 144: 1584-1592

[58] Ahvenainen $\mathrm{T}$, Lehtonen $\mathrm{HJ}$, Lehtonen $\mathrm{R}$ et al. Mutation screening of fumarate hydratase by multiplex ligation-dependent probe amplification: detection of exonic deletion in a patient with leiomyomatosis and renal cell cancer. Cancer Genet Cytogenet 2008; 183: 83-88

[59] Pavlovich CP, Schmidt LS. Searching for the hereditary causes of renal-cell carcinoma. Nat Rev Cancer 2004; 4: 381-393

[60] Gupta GN, Peterson J, Thakore KN et al. Oncological outcomes of partial nephrectomy for multifocal renal cell carcinoma greater than 4 Cm. J Urol 2010; 184: 59-63 